\title{
El ibuprofeno y la indometacina fueron similares en el tratamiento del conducto arterioso permeable en prematuros
}

\author{
A comparison of Ibuprofen and Indomethacin for closure of Patent Ductus Arteriosus.
}

Van Overmeire B, Smets K, Lecoutere D et al. N Engl J Med 2000; 343: 674-681

\section{Objetivo}

Comparar la eficacia del ibuprofeno y de la indometacina en e tratamiento del conducto arterioso permeable (CAP) en recién nacidos prematuros con síndrome de dificultad respiratoria.

\section{Diseño}

Ensayo clínico aleatorizado doble ciego

\section{Lugar}

Estudio multicéntrico realizado en cinco Unidades de Cuidados Intensivos Neonatales de Bélgica.

\section{Pacientes}

Neonatos $(n=148)$ con edad gestacional menor o igual a 32 semanas y edad postnatal entre dos y cuatro días, evidencia ecocardiográfica de CAP y diagnóstico de síndrome de dificultad respiratoria en asistencia respiratoria.

\section{Intervención}

Los pacientes recibieron, en forma aleatorizada, tres dosis de indometacina ( $0.2 \mathrm{mg} / \mathrm{kg}$ cada 12 horas) o tres dosis de ibuprofeno (dosis inicial de $10 \mathrm{mg} / \mathrm{kg}$, seguida en intervalos de $24 \mathrm{~h}$ por dos dosis de $5 \mathrm{mg} / \mathrm{kg}$ ). De no producirse el cierre del CAP y permanecer el paciente en asistencia respiratoria, se administraba en forma abierta una nueva serie de indometacina (en ambos grupos). Si esta terapia fallaba y el paciente permanecía en ventilación mecánica o si había una contraindicación para el segundo tratamiento farmacológico, se indicaba el cierre quirúrgico del conducto.

\section{Medición de resultados principales}

El resultado principal fue la tasa de cierre del conducto, confirmada por ecocardiografía.

\section{Resultados principales}

La tasa de cierre del CAP fue similar con los dos tratamientos. En el grupo ibuprofeno, el cierre se produjo en un $70 \%$ de los neonatos, mientras que en el grupo indometacina, en un $66 \%$ (RR 1.06, IC95\% 0.85-1.31; $p=0.41$ ). El número de pacientes que requirieron cierre quirúrgico fue similar en los dos grupos (ibuprofeno $14 \%$, indometacina $12 \% ; p=0.81$ ).

Un menor número de neonatos en el grupo ibuprofeno desarrolló oliguria en comparación con el grupo indometacina $(6.7 \%$ vs $19 \%, p=0.03$ ). No hubo diferencias con respecto a otros efectos adversos o complicaciones.

Conclusiones: El tratamiento del CAP con ibuprofeno en prematuros con síndrome de dificultad respiratoria es tan eficaz como la indometacina y se asocia a menor riesgo de desarrollo de oliguria.

\section{Comentario}

El texto completo de este trabajo fue publicado por el "New England" en Internet como "Early Release" en Julio de 2000 y publicado en Septiembre en papel. Este recurso se reserva para los artículos que, a juicio de los editores, tengan implicancias clínicas trascendentes y merezcan ser dadas a conocer sin demora. El CAP constituye un problema serio dentro de la salud de los recién nacidos prematuros. Su prevalencia es cercana al 30\% en menores de $1500 \mathrm{~g}$, aunque en los niños más pequeños con síndrome de dificultad respiratoria alcanza el $70 \%$. Su presencia se ha asociado a una mayor morbimortalidad neonatal (hemorragia pulmonar, enterocolitis necrotizante, enfermedad pulmonar crónica) ${ }^{1}$. El tratamiento habitual se realiza con indometacina, con una tasa de cierre en la primera serie de aproximadamente $70 \%$. Sin embargo, su utilización no está exenta de riesgos, ya que afecta la perfusión cerebral, gastrointestinal y renal, además de condicionar plaquetopenia. Vale mencionar, no obstante, que estos efectos adversos son en general leves y reversibles.

Estudios previos evaluaron la posibilidad de utilizar ibuprofeno como alternativa, con resultados alentadores2, 3. El presente trabajo agrega evidencia de mayor peso a favor del uso de ibuprofeno, ya que se trata de un estudio clínico aleatorizado con un mayor número de pacientes.

Existen ciertas observaciones que merecen ser mencionadas. E estudio cumple con los lineamientos adecuados de un ensayo clínico aleatorizado en cuanto a validez, importancia de los hallazgos y generalizabilidad de los mismos. Hay un detalle sin embargo que creo importante señalar. Vale aclarar que en este trabajo, el cálculo del tamaño muestral se realizó queriendo detectar una diferencia de al menos un $20 \%$ en la tasa de cierre entre los grupos. Esto es poco realista de acuerdo a los estudios previos, en ninguno de los cuales se habían detectado diferen- cias en la tasa de cierre de conducto arterioso entre el ibuprofeno y la indometacina. Por este motivo, aunque no parece haber diferencias clínicamente relevantes, podría haber diferencias más pequeñas no detectadas en este estudio. Para detectar diferencias más pequeñas, si las hubiere, necesitará incluir más pacientes 4 .

Creo importante subrayar que la eficacia comparable del ibuprofeno y la indometacina en recién nacidos debe restringirse al tratamiento del CAP. La indometacina, administrada dentro de las primeras doce horas de vida, disminuye la incidencia de hemorragia intracraneana en prematuros menores de $1250 \mathrm{~g}^{5}$. Es posible que el efecto diferente de la indometacina y el ibuprofeno sobre el lecho vascular cerebral haga al ibuprofeno ineficaz para ese fin.

Si bien no pareciera que los hallazgos de este artículo sean tan revolucionarios o trascendentes, la alternativa de una droga mejor tolerada es de consideración. Recordemos que los recién nacidos prematuros presentan una inmadurez que los convierte en susceptibles de múltiples complicaciones. Pero hay un aspecto adicional que juzgo importante para nuestro país. En Argentina no disponemos de la formulación farmacéutica de indometacina de $1 \mathrm{mg} / \mathrm{ml}$ (INDOCIN $\otimes$ ) por lo que debemos usar el IM 75® de $50 \mathrm{mg}$, lo que obliga a diluciones que pueden comprometer la administración de la dosis correcta. En este sentido, a la posibilidad de utilizar una medicación alternativa con probable igual eficacia y menores efectos adversos se le suma la ventaja de la seguridad de su administración.

En resumen, a pesar de ciertas reservas con respecto al diseño del estudio, los hallazgos del presente trabajo permiten considerar al ibuprofeno como una droga alternativa para el tratamiento inicial del conducto arterioso permeable a partir del tercer día de vida en prematuros con síndrome de dificultad respiratoria

\section{Dr. Gonzalo Mariani [ División Neonatología. Hospital Italiano de Buenos Aires ]}

\section{Referencias}

1. Clyman RI. Conducto arterioso permeable en el recién nacido prematuro. En Tratado de Neonatología de Avery. Taeusch HW \& Ballard RA (eds). Ediciones Harcourt 2000.

2. Varvarigou A. Early ibuprofen administration to prevent patent ductus arteriosus in premature newborn infants. A JAMA 1996 Feb 21;275(7):539-44

3. Van Overmeire B et al. Treatment of patent ductus arteriosus with Ibuprofen. Arch Dis Child 1997; 76: F179-F184

4. Landow L. Current issues in clinical trial design: superiority versus equivalency studies. Anesthesiology 2000 Jun;92(6):1814-20

5. Ment LR et al. Low-dose indomethacin and prevention of intraventricular hemorrhage: a multicenter randomized trial. Pediatrics 1994 Apr;93(4):543-50 